DESY 95-033

February 1995

\title{
Dijet Cross Sections in Photoproduction at HERA
}

\author{
ZEUS Collaboration
}

\begin{abstract}
Dijet production by almost real photons has been studied at HERA with the ZEUS detector. Jets have been identified using the cone algorithm. A cut on $x_{\gamma}^{O B S}$, the fraction of the photon energy participating in the production of the two jets of highest transverse energy, is used to define cross sections sensitive to the parton distributions in the proton and in the photon. The dependence of the dijet cross sections on pseudorapidity has been measured for $x_{\gamma}^{O B S} \geq 0.75$ and $x_{\gamma}^{O B S}<0.75$. The former is sensitive to the gluon momentum density in the proton. The latter is sensitive to the gluon in the photon. The cross sections are corrected for detector acceptance and compared to leading order QCD calculations.
\end{abstract}


M. Derrick, D. Krakauer, S. Magill, D. Mikunas, B. Musgrave, J. Repond, R. Stanek, R.L. Talaga, H. Zhang

Argonne National Laboratory, Argonne, IL, USA ${ }^{p}$

R. Ayad ${ }^{1}$, G. Bari, M. Basile, L. Bellagamba, D. Boscherini, A. Bruni, G. Bruni, P. Bruni, G. Cara Romeo, G. Castellini' ${ }^{2}$ M. Chiarini, L. Cifarelli ${ }^{3}$, F. Cindolo, A. Contin, M. Corradi, I. Gialas,

P. Giusti, G. Iacobucci, G. Laurenti, G. Levi, A. Margotti, T. Massam, R. Nania, C. Nemoz,

F. Palmonari, A. Polini, G. Sartorelli, R. Timellini, Y. Zamora Garcia ${ }^{1}$, A. Zichichi

University and INFN Bologna, Bologna, Italy ${ }^{f}$

A. Bargende, J. Crittenden, K. Desch, B. Diekmann ${ }^{4}$, T. Doeker, M. Eckert, L. Feld, A. Frey, M. Geerts, G. Geitz ${ }^{5}$, M. Grothe, T. Haas, H. Hartmann, D. Haun ${ }^{4}$, K. Heinloth, E. Hilger,

H.-P. Jakob, U.F. Katz, S.M. Mari, A. Mass ${ }^{6}$, S. Mengel, J. Mollen, E. Paul, Ch. Rembser, R. Schattevoy ${ }^{7}$, D. Schramm, J. Stamm, R. Wedemeyer

Physikalisches Institut der Universität Bonn, Bonn, Federal Republic of Germany ${ }^{c}$

S. Campbell-Robson, A. Cassidy, N. Dyce, B. Foster, S. George, R. Gilmore, G.P. Heath, H.F. Heath, T.J. Llewellyn, C.J.S. Morgado, D.J.P. Norman, J.A. O'Mara, R.J. Tapper, S.S. Wilson, R. Yoshida

H.H. Wills Physics Laboratory, University of Bristol, Bristol, U.K. ${ }^{o}$

R.R. Rau

Brookhaven National Laboratory, Upton, L.I., USA ${ }^{p}$

M. Arneodo ${ }^{8}$, L. Iannotti, M. Schioppa, G. Susinno

Calabria University, Physics Dept.and INFN, Cosenza, Italy ${ }^{f}$

A. Bernstein, A. Caldwell, N. Cartiglia, J.A. Parsons, S. Ritz, F. Sciulli, P.B. Straub, L. Wai, S. Yang, Q. Zhu Columbia University, Nevis Labs., Irvington on Hudson, N.Y., USA ${ }^{q}$

P. Borzemski, J. Chwastowski, A. Eskreys, K. Piotrzkowski, M. Zachara, L. Zawiejski

Inst. of Nuclear Physics, Cracow, Poland ${ }^{j}$

L. Adamczyk, B. Bednarek, K. Jeleń, D. Kisielewska, T. Kowalski, E. Rulikowska-Zarębska,

L. Suszycki, J. Zając

Faculty of Physics and Nuclear Techniques, Academy of Mining and Metallurgy, Cracow, Poland ${ }^{j}$

A. Kotański, M. Przybycień

Jagellonian Univ., Dept. of Physics, Cracow, Poland ${ }^{k}$

L.A.T. Bauerdick, U. Behrens, H. Beier ${ }^{9}$, J.K. Bienlein, C. Coldewey, O. Deppe, K. Desler, G. Drews,

M. Flasiński ${ }^{10}$, D.J. Gilkinson, C. Glasman, P. Göttlicher, J. Große-Knetter, B. Gutjahr, W. Hain, D. Hasell, H. Heßling, H. Hultschig, Y. Iga, P. Joos, M. Kasemann, R. Klanner, W. Koch, L. Köpke ${ }^{11}$, U. Kötz, H. Kowalski, J. Labs, A. Ladage, B. Löhr, M. Löwe, D. Lüke, O. Mańczak, J.S.T. Ng, S. Nickel, D. Notz, K. Ohrenberg, M. Roco, M. Rohde, J. Roldán, U. Schneekloth, W. Schulz, F. Selonke, E. Stiliaris ${ }^{12}$, B. Surrow, T. Voß, D. Westphal, G. Wolf, C. Youngman, J.F. Zhou

Deutsches Elektronen-Synchrotron DESY, Hamburg, Federal Republic of Germany

H.J. Grabosch, A. Kharchilava, A. Leich, M. Mattingly, A. Meyer, S. Schlenstedt, N. Wulff

DESY-Zeuthen, Inst. für Hochenergiephysik, Zeuthen, Federal Republic of Germany

G. Barbagli, P. Pelfer

University and INFN, Florence, Italy ${ }^{f}$

G. Anzivino, G. Maccarrone, S. De Pasquale, L. Votano

INFN, Laboratori Nazionali di Frascati, Frascati, Italy ${ }^{f}$

A. Bamberger, S. Eisenhardt, A. Freidhof, S. Söldner-Rembold ${ }^{13}$, J. Schroeder ${ }^{14}$, T. Trefzger

Fakultät für Physik der Universität Freiburg i.Br., Freiburg i.Br., Federal Republic of Germany ${ }^{c}$ 
N.H. Brook, P.J. Bussey, A.T. Doyle ${ }^{15}$, J.I. Fleck, D.H. Saxon, M.L. Utley, A.S. Wilson

Dept. of Physics and Astronomy, University of Glasgow, Glasgow, U.K. ${ }^{\circ}$

A. Dannemann, U. Holm, D. Horstmann, T. Neumann, R. Sinkus, K. Wick

Hamburg University, I. Institute of Exp. Physics, Hamburg, Federal Republic of Germany ${ }^{c}$

E. Badura ${ }^{16}$, B.D. Burow ${ }^{17}$, L. Hagge, E. Lohrmann, J. Mainusch, J. Milewski, M. Nakahata ${ }^{18}$, N. Pavel, G. Poelz, W. Schott, F. Zetsche

Hamburg University, II. Institute of Exp. Physics, Hamburg, Federal Republic of Germany ${ }^{c}$

T.C. Bacon, I. Butterworth, E. Gallo, V.L. Harris, B.Y.H. Hung, K.R. Long, D.B. Miller, P.P.O. Morawitz, A. Prinias, J.K. Sedgbeer, A.F. Whitfield

Imperial College London, High Energy Nuclear Physics Group, London, U.K. ${ }^{\circ}$

U. Mallik, E. McCliment, M.Z. Wang, S.M. Wang, J.T. Wu, Y. Zhang

University of Iowa, Physics and Astronomy Dept., Iowa City, USA ${ }^{p}$

P. Cloth, D. Filges

Forschungszentrum Jülich, Institut für Kernphysik, Jülich, Federal Republic of Germany

S.H. An, S.M. Hong, S.W. Nam, S.K. Park, M.H. Suh, S.H. Yon

Korea University, Seoul, Korea ${ }^{h}$

R. Imlay, S. Kartik, H.-J. Kim, R.R. McNeil, W. Metcalf, V.K. Nadendla

Louisiana State University, Dept. of Physics and Astronomy, Baton Rouge, LA, USA ${ }^{p}$

F. Barreiro ${ }^{19}$, G. Cases, R. Graciani, J.M. Hernández, L. Hervás ${ }^{19}$, L. Labarga ${ }^{19}$, J. del Peso, J. Puga, J. Terron, J.F. de Trocóniz

Univer. Autónoma Madrid, Depto de Física Teóríca, Madrid, Spain ${ }^{n}$

G.R. Smith

University of Manitoba, Dept. of Physics, Winnipeg, Manitoba, Canada ${ }^{a}$

F. Corriveau, D.S. Hanna, J. Hartmann, L.W. Hung, J.N. Lim, C.G. Matthews, P.M. Patel,

L.E. Sinclair, D.G. Stairs, M. St.Laurent, R. Ullmann, G. Zacek

McGill University, Dept. of Physics, Montréal, Québec, Canada ${ }^{a},{ }^{b}$

V. Bashkirov, B.A. Dolgoshein, A. Stifutkin

Moscow Engineering Physics Institute, Mosocw, Russia ${ }^{l}$

G.L. Bashindzhagyan, P.F. Ermolov, L.K. Gladilin, Y.A. Golubkov, V.D. Kobrin, V.A. Kuzmin, A.S. Proskuryakov, A.A. Savin, L.M. Shcheglova, A.N. Solomin, N.P. Zotov

Moscow State University, Institute of Nuclear Pysics, Moscow, Russia ${ }^{m}$

M. Botje, F. Chlebana, A. Dake, J. Engelen, M. de Kamps, P. Kooijman, A. Kruse, H. Tiecke, W. Verkerke, M. Vreeswijk, L. Wiggers, E. de Wolf, R. van Woudenberg

NIKHEF and University of Amsterdam, Netherlands ${ }^{i}$

D. Acosta, B. Bylsma, L.S. Durkin, K. Honscheid, C. Li, T.Y. Ling, K.W. McLean ${ }^{20}$, W.N. Murray, I.H. Park, T.A. Romanowski ${ }^{21}$, R. Seidlein ${ }^{22}$

Ohio State University, Physics Department, Columbus, Ohio, USA ${ }^{p}$

D.S. Bailey, G.A. Blair ${ }^{23}$, A. Byrne, R.J. Cashmore, A.M. Cooper-Sarkar, D. Daniels ${ }^{24}$,

R.C.E. Devenish, N. Harnew, M. Lancaster, P.E. Luffman ${ }^{25}$, L. Lindemann, J.D. McFall, C. Nath, V.A. Noyes, A. Quadt, H. Uijterwaal, R. Walczak, F.F. Wilson, T. Yip

Department of Physics, University of Oxford, Oxford, U.K. ${ }^{o}$

G. Abbiendi, A. Bertolin, R. Brugnera, R. Carlin, F. Dal Corso, M. De Giorgi, U. Dosselli,

S. Limentani, M. Morandin, M. Posocco, L. Stanco, R. Stroili, C. Voci

Dipartimento di Fisica dell' Universita and INFN, Padova, Italy ${ }^{f}$ 
J. Bulmahn, J.M. Butterworth, R.G. Feild, B.Y. Oh, J.J. Whitmore ${ }^{26}$

Pennsylvania State University, Dept. of Physics, University Park, PA, USA ${ }^{q}$

G. D'Agostini, G. Marini, A. Nigro, E. Tassi

Dipartimento di Fisica, Univ. 'La Sapienza' and INFN, Rome, Italy ${ }^{f}$

J.C. Hart, N.A. McCubbin, K. Prytz, T.P. Shah, T.L. Short

Rutherford Appleton Laboratory, Chilton, Didcot, Oxon, U.K. ${ }^{o}$

E. Barberis, T. Dubbs, C. Heusch, M. Van Hook, B. Hubbard, W. Lockman,

J.T. Rahn, H.F.-W. Sadrozinski, A. Seiden

University of California, Santa Cruz, CA, USA ${ }^{p}$

J. Biltzinger, R.J. Seifert, A.H. Walenta, G. Zech

Fachbereich Physik der Universität-Gesamthochschule Siegen, Federal Republic of Germany ${ }^{c}$

H. Abramowicz, G. Briskin, S. Dagan ${ }^{27}$, A. Levy ${ }^{28}$

School of Physics, Tel-Aviv University, Tel Aviv, Israel ${ }^{e}$

T. Hasegawa, M. Hazumi, T. Ishii, M. Kuze, S. Mine, Y. Nagasawa, M. Nakao, I. Suzuki, K. Tokushuku, S. Yamada, Y. Yamazaki

Institute for Nuclear Study, University of Tokyo, Tokyo, Japan ${ }^{g}$

M. Chiba, R. Hamatsu, T. Hirose, K. Homma, S. Kitamura, Y. Nakamitsu, K. Yamauchi

Tokyo Metropolitan University, Dept. of Physics, Tokyo, Japan ${ }^{g}$

R. Cirio, M. Costa, M.I. Ferrero, L. Lamberti, S. Maselli, C. Peroni, R. Sacchi, A. Solano, A. Staiano

Universita di Torino, Dipartimento di Fisica Sperimentale and INFN, Torino, Italy ${ }^{f}$

M. Dardo

II Faculty of Sciences, Torino University and INFN - Alessandria, Italy ${ }^{f}$

D.C. Bailey, D. Bandyopadhyay, F. Benard, M. Brkic, M.B. Crombie, D.M. Gingrich ${ }^{29}$, G.F. Hartner, K.K. Joo, G.M. Levman, J.F. Martin, R.S. Orr, C.R. Sampson, R.J. Teuscher

University of Toronto, Dept. of Physics, Toronto, Ont., Canada ${ }^{a}$

C.D. Catterall, T.W. Jones, P.B. Kaziewicz, J.B. Lane, R.L. Saunders, J. Shulman

University College London, Physics and Astronomy Dept., London, U.K. ${ }^{\circ}$

K. Blankenship, J. Kochocki, B. Lu, L.W. Mo

Virginia Polytechnic Inst. and State University, Physics Dept., Blacksburg, VA, USA ${ }^{q}$

W. Bogusz, K. Charchuła, J. Ciborowski, J. Gajewski, G. Grzelak, M. Kasprzak, M. Krzyżanowski,

K. Muchorowski, R.J. Nowak, J.M. Pawlak, T. Tymieniecka, A.K. Wróblewski, J.A. Zakrzewski, A.F. Żarnecki Warsaw University, Institute of Experimental Physics, Warsaw, Poland ${ }^{j}$

M. Adamus

Institute for Nuclear Studies, Warsaw, Poland ${ }^{j}$

Y. Eisenberg ${ }^{27}$, U. Karshon ${ }^{27}$, D. Revel ${ }^{27}$, D. Zer-Zion

Weizmann Institute, Nuclear Physics Dept., Rehovot, Israel ${ }^{d}$

I. Ali, W.F. Badgett, B. Behrens, S. Dasu, C. Fordham, C. Foudas, A. Goussiou, R.J. Loveless, D.D. Reeder, S. Silverstein, W.H. Smith, A. Vaiciulis, M. Wodarczyk

University of Wisconsin, Dept. of Physics, Madison, WI, USA ${ }^{p}$

T. Tsurugai

Meiji Gakuin University, Faculty of General Education, Yokohama, Japan

S. Bhadra, M.L. Cardy, C.-P. Fagerstroem, W.R. Frisken, K.M. Furutani, M. Khakzad, W.B. Schmidke

York University, Dept. of Physics, North York, Ont., Canada ${ }^{a}$ 
${ }^{1}$ supported by Worldlab, Lausanne, Switzerland

2 also at IROE Florence, Italy

${ }^{3}$ now at Univ. of Salerno and INFN Napoli, Italy

${ }^{4}$ now a self-employed consultant

${ }^{5}$ on leave of absence

${ }^{6}$ now at Institut für Hochenergiephysik, Univ. Heidelberg

7 now at MPI Berlin

8 now also at University of Torino

${ }^{9}$ presently at Columbia Univ., supported by DAAD/HSPII-AUFE

10 now at Inst. of Computer Science, Jagellonian Univ., Cracow

11 now at Univ. of Mainz

12 supported by the European Community

13 now with OPAL Collaboration, Faculty of Physics at Univ. of Freiburg

${ }^{14}$ now at SAS-Institut GmbH, Heidelberg

15 also supported by DESY

16 now at GSI Darmstadt

17 also supported by NSERC

18 now at Institute for Cosmic Ray Research, University of Tokyo

19 on leave of absence at DESY, supported by DGICYT

20 now at Carleton University, Ottawa, Canada

${ }^{21}$ now at Department of Energy, Washington

22 now at HEP Div., Argonne National Lab., Argonne, IL, USA

23 now at RHBNC, Univ. of London, England

${ }^{24}$ Fulbright Scholar 1993-1994

25 now at Cambridge Consultants, Cambridge, U.K.

${ }^{26}$ on leave and partially supported by DESY 1993-95

27 supported by a MINERVA Fellowship

28 partially supported by DESY

${ }^{29}$ now at Centre for Subatomic Research, Univ.of Alberta, Canada and TRIUMF, Vancouver, Canada

a supported by the Natural Sciences and Engineering Research Council of Canada (NSERC)

$b$ supported by the FCAR of Québec, Canada

c supported by the German Federal Ministry for Research and Technology (BMFT)

$d$ supported by the MINERVA Gesellschaft für Forschung GmbH, and by the Israel Academy of Science

$e \quad$ supported by the German Israeli Foundation, and by the Israel Academy of Science

$f \quad$ supported by the Italian National Institute for Nuclear Physics (INFN)

$g \quad$ supported by the Japanese Ministry of Education, Science and Culture (the Monbusho) and its grants for Scientific Research

$h$ supported by the Korean Ministry of Education and Korea Science and Engineering Foundation

$i \quad$ supported by the Netherlands Foundation for Research on Matter (FOM)

$j$ supported by the Polish State Committee for Scientific Research (grant No. SPB/P3/202/93) and the Foundation for Polish- German Collaboration (proj. No. 506/92)

$k \quad$ supported by the Polish State Committee for Scientific Research (grant No. PB 861/2/91 and No. 22372 9102, grant No. PB 223769102 and No. PB 20092 9101)

$l$ partially supported by the German Federal Ministry for Research and Technology (BMFT)

$m$ supported by the German Federal Ministry for Research and Technology (BMFT), the Volkswagen Foundation, and the Deutsche Forschungsgemeinschaft

$n$ supported by the Spanish Ministry of Education and Science through funds provided by CICYT

$o$ supported by the Particle Physics and Astronomy Research Council

$p \quad$ supported by the US Department of Energy

$q \quad$ supported by the US National Science Foundation 


\section{Introduction}

At the high energies available at HERA, interactions between almost real photons (of virtuality $Q^{2} \approx 0$ ) and protons produce jets of high transverse energy [1, 2, 3, 4, 5]. The presence of a 'hard' energy scale means that perturbative QCD calculations of event properties can be confronted with experiment. At leading order (LO) two processes are responsible for jet production. The photon may interact directly with a parton in the proton (Fig. 1a), or it may first fluctuate into an hadronic state (Fig. 1 b). In the first case, known as the direct contribution, the full energy of the photon participates in the interaction with a parton in the proton and the fraction of the photon momentum $\left(x_{\gamma}\right)$ participating in the hard process is equal to one. The final state of the direct process consists of two jets, the proton remnant and the scattered electron. In the second case, known as the resolved contribution, the photon acts as a source of partons which then scatter off partons in the proton and the fraction of the photon momentum participating in the hard process is less than one. The final state in this case includes a photon remnant, continuing in the original photon direction, in addition to two jets, the proton remnant and the scattered electron. At higher orders this simple distinction between direct and resolved is no longer precisely defined.

With a cut on jet transverse energy of $E_{T}^{j e t}>6 \mathrm{GeV}$, direct photon events probe the parton distributions in the proton down to $x_{p} \approx 2 \times 10^{-3}$, where $x_{p}$ is the fraction of the proton's momentum entering into the hard process. This process is directly sensitive to the gluon distribution in the proton, and complements indirect extractions in the same $x_{p}$ range [6] using the measurement of $F_{2}$ in deep inelastic scattering (DIS). The $x_{p}$ values sampled by the resolved photon contribution are typically higher than those of the direct contribution, lying in a region where the proton parton distributions are constrained by DIS data. Resolved photon processes are directly sensitive to the photon parton distributions, particularly the gluon distribution in the photon, down $x_{\gamma} \approx 0.06$. This is not the case in the measurement of $F_{2}^{\gamma}$ in $\gamma \gamma$ interactions at $e^{+} e^{-}$colliders, where highly virtual photons are used to probe almost real photons [7]. In collisions between two almost real photons, however, the measurement of jet cross sections has recently shown potential to constrain the gluon distribution in the photon [8, 9].

In this paper we separate the direct and resolved photon contributions to jet production, and present dijet differential ep cross sections which are sensitive to the gluon distributions in the proton and photon respectively. In describing the cross sections to be measured, particular attention is paid to the way in which direct and resolved processes are defined. Differential $e p$ cross sections are presented as a function of jet pseudorapidity for direct and resolved photon processes and compared to available LO QCD calculations.

\section{Definition of cross sections}

We measure the cross section for dijet photoproduction, $e p \rightarrow e \gamma p \rightarrow e X$, for events in which $X$ contains at least two jets of $E_{T}^{j e t}>6 \mathrm{GeV}$. In this experiment, photoproduction events are defined by demanding that the electron is scattered at small angles and does not emerge from the beam pipe. This requirement corresponds approximately to a cut of $Q^{2}<4 \mathrm{GeV}^{2}$, giving a median $Q^{2}$ of $\sim 10^{-3} \mathrm{GeV}^{2}$ 喵. The cross section measured is $d \sigma / d \bar{\eta}$, where $\bar{\eta}=\frac{1}{2}\left(\eta_{1}+\eta_{2}\right)$ is the average pseudorapidity of the two jets of highest transverse energy, with the requirement

\footnotetext{
${ }^{1}$ The $z$ axis is defined to lie along the proton direction, and $\eta=-\ln \left(\tan \frac{\theta}{2}\right)$ where $\theta$ is the angle between the jet and the $z$ axis.
} 
that $|\Delta \eta|=\left|\eta_{1}-\eta_{2}\right|<0.5$. The cross section is measured for $0.2<y<0.8$, where $y=E_{\gamma} / E_{e}$ is the fraction of the initial electron energy $\left(E_{e}\right)$ carried by the almost real photon with energy $E_{\gamma}$. This $y$ interval corresponds to $\gamma p$ centre-of-mass energies $\left(W_{\gamma p}\right)$ in the range $132 \mathrm{GeV}$ $<W_{\gamma p}<265 \mathrm{GeV}$.

The possibility of experimentally separating samples of direct and resolved photon events was demonstrated in [4]. However, as the simple definition of resolved and direct photoproduction is only unambiguous at leading order, it is important to find a definition which is both calculable to all orders and measurable.

For two-to-two parton scattering in LO QCD, energy and momentum conservation give the fraction of the photon energy involved in the hard scatter as

$$
x_{\gamma}^{L O}=\frac{\sum_{\text {partons }} E_{T}^{\text {parton }} e^{-\eta^{\text {parton }}}}{2 y E_{e}},
$$

where $y E_{e}$ is the initial photon energy and the sum is over the two final state partons. For direct photon events, $x_{\gamma}^{L O}=1$. Since it is not possible to measure partons, we define an observable in terms of jets which is analogous to $x_{\gamma}^{L O}$. This observable, called $x_{\gamma}^{O B S}$, is the fraction of the photon's momentum participating in the production of the two highest $E_{T}^{\text {jet }}$ jets. The explicit definition is,

$$
x_{\gamma}^{O B S}=\frac{\sum_{j e t s} E_{T}^{j e t} e^{-\eta^{j e t}}}{2 y E_{e}},
$$

where now the sum runs over the two jets of highest $E_{T}^{j e t}$. In the $x_{\gamma}^{O B S}$ distribution thus obtained, the LO direct and resolved processes populate different regions, with the direct processes concentrated at high values of $x_{\gamma}^{O B S}$. The peak arising from the direct contribution will not necessarily lie exactly at $x_{\gamma}^{O B S}=1$ due to higher order effects and/or hadronisation, but will still correspond to the kinematic region where most or all of the energy of the photon is available to probe the proton. The relationship between the definitions of $x_{\gamma}^{O B S}$ and $x_{\gamma}^{L O}$ is dependent upon the hadronisation and showering models used to describe the final state and the assumptions made in the calculation of the hard process, as $x_{\gamma}^{L O}$ becomes ambiguous even at next-to-leading order (NLO). For these reasons, we will present hadronic jet cross sections and will not use Monte Carlo models to correct back to parton kinematics. For the purposes of this paper, the separation between direct and resolved photoproduction is defined by a cut on $x_{\gamma}^{O B S}$. Other model-independent definitions for separating resolved and direct photon processes have been suggested, often using a cut on the energy in a cone around the photon direction 10 . We have chosen the definition based upon $x_{\gamma}^{O B S}$ because it depends only upon the measurement of $y, E_{T}^{j e t}$ and the $\eta$ 's of the jets, without the need to introduce further variables.

The $|\Delta \eta|$ cut ensures that for the $\bar{\eta}$ bins in which the cross sections are measured, both jets lie well within the acceptance of the ZEUS detector, without imposing additional cuts at high and low $\eta^{\text {jet }}$. Applying this cut has two further benefits, which can be seen as follows. When both jets are at equal $\eta$ and equal $E_{T}^{\text {jet }}, \sqrt{\hat{s}}=2 E_{T}^{\text {jet }}$ and so the range of possible choices of scale in theoretical calculations is reduced. More importantly, by rewriting the expression for $x_{\gamma}^{O B S}$ in terms of $|\Delta \eta|$ and $\bar{\eta}$, and assuming the jets to have equal transverse energy, one obtains 


$$
x_{\gamma}^{O B S}=\frac{E_{T}^{j e t} e^{-\bar{\eta}}}{y E_{e}} \cosh \frac{\Delta \eta}{2} .
$$

A similar expression can be written for the proton,

$$
x_{p}^{O B S}=\frac{E_{T}^{j e t} e^{\bar{\eta}}}{E_{p}} \cosh \frac{\Delta \eta}{2},
$$

where $E_{p}$ is the incident proton energy. When the jets are at equal pseudorapidities the hyperbolic cosine term takes its minimum value of unity. Thus the minimum available $x$ values are probed for a given $E_{T}^{j e t}$ and $\bar{\eta}$, and there is a strong correlation between $\bar{\eta}$ and $x_{p}^{O B S}$ in the direct cross section and between $\bar{\eta}$ and $y x_{\gamma}^{O B S}$ in the resolved cross section [11]. Applying the cut $|\Delta \eta|<0.5$ brings us close to this situation.

\section{The ZEUS detector and beam conditions}

Details of the ZEUS detector have been described elsewhere [12]. The primary components used in this analysis are the calorimeter and the tracking detectors. The uranium-scintillator calorimeter [13] covers about $99.7 \%$ of the total solid angle and is subdivided into electromagnetic and hadronic sections with cell size, respectively, of $5 \times 20 \mathrm{~cm}^{2}\left(10 \times 20 \mathrm{~cm}^{2}\right.$ in the rear calorimeter i.e. the electron direction), and $20 \times 20 \mathrm{~cm}^{2}$. The calorimeter has an equal response to electrons and hadrons within $3 \%$ and the energy resolution achieved in test beams, in terms of the energy $E$ in $\mathrm{GeV}$, is $\sigma / E=18 \% / \sqrt{E}$ for electrons and $\sigma / E=35 \% / \sqrt{E}$ for hadrons. The timing resolution of a calorimeter cell is better than $\sigma_{t}=1.5 / \sqrt{E} \oplus 0.5 \mathrm{~ns}$.

The tracking system consists of a vertex detector (VXD) [14] and a central tracking chamber (CTD) 15 enclosed in a $1.43 \mathrm{~T}$ solenoidal magnetic field. The interaction vertex is measured with a resolution along (transverse to) the beam direction of $0.4(0.1) \mathrm{cm}$.

To allow a precise measurement of the luminosity via the electron-proton Bremsstrahlung process, electron and photon lead-scintillator calorimeters have been installed inside the HERA tunnel, subtending small angles from the interaction vertex [16]. The small-angle electron calorimeter is also used to tag a subsample of photoproduction events in the approximate range $10^{-8} \mathrm{GeV}^{2}<Q^{2}<10^{-2} \mathrm{GeV}^{2}$.

In 1993 HERA operated with 84 colliding bunches of $820 \mathrm{GeV}$ protons and $26.7 \mathrm{GeV}$ electrons with typical beam currents around $10 \mathrm{~mA}$ and a luminosity of $0.6 \times 10^{30} \mathrm{~cm}^{-2} \mathrm{~s}^{-1}$. From these colliding bunches ZEUS collected a total integrated luminosity of approximately $0.55 \mathrm{pb}^{-1}$. Additional unpaired electron and proton bunches circulated to allow monitoring of background from beam-gas interactions. 


\section{Data selection and jet finding}

ZEUS data acquisition uses a three level trigger system. During the 1993 data taking period, we have selected events which were triggered at the first level on regional or transverse energy sums in the uranium calorimeter. Events were also triggered on a coincidence of an electron measured in the small-angle electron calorimeter and an energy deposit in the rear calorimeter. These events were used to check the efficiency of the calorimeter-only triggers used in the cross section calculation.

At the second level trigger, cuts on calorimeter timing were used to remove events caused by interactions between the proton beam and residual gas in the beam pipe in front of the calorimeter [17]. The efficiency of the combination of the first and second level triggers to select events in the kinematic region of our cross sections has been determined to be greater than $98 \%$.

At the third level trigger, tighter timing cuts were made to reject events arising from proton beam-gas interactions. Cosmic ray events were vetoed using information from the tracking chambers and calorimeter. Events with no vertex found by the central tracking chambers, or with a vertex found at $z<-75 \mathrm{~cm}$ were rejected. The measured transverse energy outside a $10^{\circ}$ cone around the forward beampipe $\left(E_{T}^{c o n e}\right)$ was calculated, making use of the vertex information from the tracking detectors and associating the energy in a cell with the geometric centre of that cell. Events with $E_{T}^{\text {cone }}<12 \mathrm{GeV}$ were rejected. After these triggers, 470,000 events remained.

For the final analysis, more stringent cuts using calorimeter timing and tracking information are made to further reduce the background from cosmic rays and beam-gas interactions. Two additional cuts are made, based upon different measurements of $y$ [四:

1. Events with an electron candidate in the uranium calorimeter are removed if $E_{e}^{\prime}$ is greater than $5 \mathrm{GeV}$, and if the electron gives a measured $y_{e}=1-\frac{E_{e}^{\prime}}{2 E_{e}}\left(1-\cos \theta_{e}^{\prime}\right)<0.7$, where $E_{e}^{\prime}$ and $\theta_{e}^{\prime}$ are the energy and angle of the scattered electron.

2. A cut is made on the Jacquet-Blondel measurement of $y, y_{J B}=\sum_{i}\left(E_{i}-E_{z i}\right) / 2 E_{e}$, where $E_{z i}=E_{i} \cos \left(\theta_{i}\right)$, and $E_{i}$ and $\theta_{i}$ are the energy and polar angle of the calorimeter cell. The angle is determined using the measured $z$-vertex of the event. It is assumed that the scattered electron is not seen in the uranium calorimeter and so the sum runs over all calorimeter cells. For any remaining events for which the scattered electron did enter the uranium calorimeter and either was not identified or gave $y_{e}$ above 0.7 , the value of $y_{J B}$ under this assumption will be near one. Proton beam-gas events will have low values of $y_{J B}$. To further reduce contamination from both these sources, we demand that $0.15<y_{J B}<0.7$, which is an estimator of the actual $y$ interval of $0.2<y<0.8$, as determined from studies of the energy loss in inactive material in front of the uranium calorimeter.

For the surviving events, jet finding is performed on all cells of the uranium calorimeter using a cone algorithm [18]. The cone radius of the jet finding algorithm is defined such that

$R=\sqrt{\Delta \eta_{\text {cell }}^{2}+\Delta \phi_{\text {cell }}^{2}}=1$. The algorithm searches in pseudorapidity-azimuth $\left(\eta_{\text {cell }}-\phi_{\text {cell }}\right)$ space for the cone containing the highest summed $E_{T}$, removes the cells in this cone and continues 
the search for the next highest $E_{T}$ cone. If the summed transverse energy of the calorimeter cells within a cone $\left(E_{T}^{c a l}\right)$ is greater than $5 \mathrm{GeV}$, and if the $E_{T}$ weighted pseudorapidity of the centre of the cone lies within $-1.125<\eta^{\text {cal }}<1.875$, then the cells inside this cone are selected as a jet. The $E_{T}^{c a l}$ threshold of $5 \mathrm{GeV}$ is to compensate for energy losses from inactive material in front of the calorimeter. This energy loss is corrected for in the determination of the cross sections. This $\eta$ range is dictated by the available statistics and by the necessity to remain in the central region of the calorimeter. Events are selected if they have at least two jets satisfying these cuts.

After these cuts a sample of 12,100 photoproduction events remains. This is reduced to a final sample of 4,000 events after a cut on the value of $\Delta \eta$ reconstructed from the two jets of highest transverse energy found in the calorimeter $\left(\left|\Delta \eta^{c a l}\right|<0.5\right)$. The backgrounds from cosmic rays and beam-gas interactions are $0.6 \%$ and $1.0 \%$, respectively, as determined from the number of surviving events originating from empty bunch crossings and unpaired proton bunches. The contamination from events with a scattered electron in the uranium calorimeter is found to be around 2.5\% from studies using DIS Monte Carlo events. Studies using the independently selected sample for which the scattered electron is seen in the small angle electron calorimeter and for which lower energy thresholds in the uranium calorimeter are applied, show that the combined efficiency of the first level trigger and the $E_{T}^{c o n e}$ cut is greater than $90 \%$ for events with two jets of $E_{T}^{c a l}>5 \mathrm{GeV}$.

\section{$5 \quad$ Resolution of kinematic variables}

In order to estimate how well jets and energies are reconstructed in the ZEUS detector, and to study the efficiency of the data selection cuts, we have used the HERWIG 5.7 19] and PYTHIA 5.6 [20] event generators in conjunction with a detailed simulation of the ZEUS detector and triggers. The simulated integrated luminosity used is greater than that of the data. As shown in [4], these generators are able to provide a reasonable description of the data with the minimum $\hat{p}_{T}$ of a hard scatter set to $2.5 \mathrm{GeV}$ and this value was used in the current implementations. The parton distribution sets used were MRSD_ 21] for the proton and GRV LO for the photon [22]. For each generator (HERWIG and PYTHIA), samples of direct and resolved photoproduction events were combined according to the generated cross sections. The Monte Carlo simulations are only used to correct for detector acceptance and smearing.

The experimental shifts and resolutions predicted by the Monte Carlo simulations were obtained by comparing the true variables with the reconstructed values in the simulated detector. The measured value of $x_{\gamma}^{O B S}$ is called $x_{\gamma}^{c a l}$ and is calculated by inserting $E_{T}^{c a l}, \eta^{c a l}$, and $y_{J B}$ into Equation (2). For the jet variables (including $x_{\gamma}^{O B S}$ ), the true variables are calculated by performing jet finding on the final state particles generated by the HERWIG or PYTHIA program before detector simulation. The reconstructed value of $y\left(y_{J B}\right)$ is systematically lower than the true value by an average of 0.08 , and has a resolution of 0.06 . The measurements of $\eta, \bar{\eta}, \Delta \eta$ and $x_{\gamma}^{O B S}$ exhibit no systematic shift and have resolutions of $0.06(0.07), 0.05$ (0.06), 0.10 (0.13) and 0.06 (0.07) respectively, for HERWIG (PYTHIA). The reconstructed value of $E_{T}^{j e t}$ is systematically lower than the true value by $15 \%(16 \%)$ for the HERWIG (PYTHIA) simulation with a resolution of $12 \%$.

The description of the energy response of the uranium calorimeter in the Monte Carlo simulation has been checked for events with an electron measured in the small-angle electron calorimeter. 
The photon energy calculated from $E_{\gamma}=E_{e}-E_{e}^{\prime}$ was compared with the value reconstructed from the calorimeter variable $y_{J B}\left(E_{\gamma}^{c a l}=y_{J B} E_{e}\right)$. In addition, for jets in the central region of the calorimeter, the measurement of the transverse energy available from the ZEUS tracking detectors has been used to check the simulation of the calorimeter energy response [5]. The transverse energy of jets in the forward region of the calorimeter (outside the acceptance of the ZEUS central tracking detector) has been compared with the $E_{T}^{\text {cal }}$ of these central jets. From these investigations we conclude that the Monte Carlo description of the energy response of the calorimeter to jets of hadrons is correct to within a possible overestimation of the energy of $5 \%$.

The effects of discrepancies between the Monte Carlo simulations and the data on the measurement of the cross sections are estimated by varying the selection cuts made on the reconstructed kinematic variables, both in the data and the simulated sample and are included in the systematic errors on the final cross section values.

\section{Results and discussion}

Fig. 2a shows the distribution of $x_{\gamma}^{\text {cal }}$ for the final data sample entering into the cross section measurements. There is a clear peak at high values of $x_{\gamma}^{\text {cal }}$ which indicates the presence of direct type processes. The shape of this uncorrected $x_{\gamma}^{O B S}$ distribution and the position of the peak are insensitive to the problem of calorimeter energy response because of the presence of energy terms in both the numerator and denominator of Equation (21). The distributions from PYTHIA and HERWIG are also shown where the Monte Carlo curves have been normalised to fit the direct peak in the data. Although the shape of the direct peak is reasonably reproduced, the Monte Carlo simulations fail to describe the rise seen in the data at low $x_{\gamma}^{\text {cal }}$. This effect was also seen in [4]. In the same Fig., the LO direct contribution from HERWIG is shown separately,

and indicates that defining the direct process with a cut on $x_{\gamma}^{O B S}$ of 0.75 corresponds well to the LO definition of direct photon processes as implemented in the simulations.

Fig. 2 $\mid \mathrm{b}$ shows the uncorrected transverse energy flow per jet $1 / N d E_{T} / d \delta \eta$ around the jet axis for events with $x_{\gamma}^{c a l} \geq 0.75$. For this class of events, both HERWIG and PYTHIA reproduce the data distribution well. The same distribution is shown in Fig. 20 for events with $x_{\gamma}^{\text {cal }}<0.75$. In this case both simulations fail to describe the transverse energy flow in the forward region, as was also observed for the jets in the inclusive jet cross sections in [3] and [5].

\subsection{Direct photon dijet cross section}

The direct photon cross section was evaluated from our data by applying acceptance correction factors obtained using the Monte Carlo simulations. Events were selected by cuts on the reconstructed kinematic quantities as outlined in Section 4 and a cut of $x_{\gamma}^{c a l} \geq 0.75$. For these cuts, we have evaluated, bin by bin, the acceptance correction for the measurement of the cross section $d \sigma / d \bar{\eta}$ as defined by the kinematic variables described in Section 2 with a cut of $x_{\gamma}^{O B S} \geq 0.75$. The efficiency and purity are evaluated for bins of width 0.25 , with centres in the range $-0.75<\bar{\eta}<1.0$, using the simulation of the ZEUS detector in conjunction with events from the HERWIG and PYTHIA generators. 


\begin{tabular}{|r|c|c|c|c|}
\hline $\bar{\eta}$ & $\begin{array}{c}d \sigma / d \bar{\eta} \\
(\mathrm{nb})\end{array}$ & $\begin{array}{c}\text { Statistical } \\
\text { Error }(\mathrm{nb})\end{array}$ & $\begin{array}{c}\text { Systematic } \\
\text { Error }(\mathrm{nb})\end{array}$ & $\begin{array}{c}\text { Energy Scale Uncertainty } \\
(\mathrm{nb})\end{array}$ \\
\hline-0.75 & 0.63 & 0.08 & 0.16 & 0.08 \\
-0.50 & 1.31 & 0.13 & 0.10 & 0.19 \\
-0.25 & 1.32 & 0.14 & 0.08 & 0.20 \\
0.00 & 1.37 & 0.11 & 0.06 & 0.24 \\
0.25 & 1.29 & 0.11 & 0.17 & 0.15 \\
0.50 & 0.88 & 0.10 & 0.17 & 0.16 \\
0.75 & 0.50 & 0.06 & 0.27 & 0.06 \\
1.00 & 0.23 & 0.04 & 0.17 & 0.05 \\
\hline
\end{tabular}

Table 1: $d \sigma / d \bar{\eta}$ for $e p \rightarrow e X+2$ (or more) jets, $|\Delta \eta|<0.5, E_{T}^{j e t}>6 \mathrm{GeV}, 0.2<y<0.8$, $Q^{2}<4 \mathrm{GeV}^{2}, x_{\gamma}^{O B S} \geq 0.75$

The efficiency is a slowly varying function of $\bar{\eta}$ and is around 50\%, falling to $35 \%$ in the lowest $\bar{\eta}$ bin. The purity in each bin is approximately independent of $\bar{\eta}$ and around $60 \%$ and depends mostly on migrations across the $E_{T}^{\text {jet }}$ cut. Taking the ratio of these gives an acceptance correction factor which averages around 1.3 and rises to around 1.5 in the lowest $\bar{\eta}$ bin. This correction accounts for all detector effects and migrations. We correct back to the final state particles and no subtraction of jet pedestal energy (i.e. possible transverse energy around the jet direction which is not associated with the hard subprocess) is performed. The differential cross section $d \sigma / d \bar{\eta}$ for $x_{\gamma}^{O B S} \geq 0.75$ and the kinematic range indicated in the figure caption, is shown in Table 1 and in Fig. 3 where the cross section value is plotted in the centre of the bins.

The systematic uncertainties in the measurement of the differential cross section have been estimated by repeating the acceptance correction with both HERWIG and PYTHIA, and by varying the cuts made on the reconstructed quantities, and by using different parton distribution sets in the simulations. In addition, the bin by bin correction procedure was checked against the results of an unfolding method based upon Bayes' theorem [23], which showed essentially the same result. The largest shift in each bin from these variations was found to be similar to the statistical error on the data, and is taken to be the total systematic error.

The systematic uncertainty arising from a possible 5\% uncertainty in the mean energies measured by the calorimeter is highly correlated between bins, and is therefore excluded from the systematic errors and shown separately as a shaded band in Fig. 3a. Also included in this shaded band is the uncertainty in the measurement of the integrated luminosity of $3.3 \%$. The shaded band represents the width of the uncertainty around each data point.

The measured direct dijet cross section is shown in Fig. 3a. The cross section is around $1.3 \mathrm{nb}$ at negative $\bar{\eta}$ values, and exhibits a sharp drop near $\bar{\eta}=0$ which arises from the cutoff on the minimum $E_{T}^{j e t}$ and the cuts on $y$. The cross section is compared to several LO QCD calculations in which the two final state partons are considered to be jets. The shape of the direct cross section differs from that of the LO QCD calculations. However, several effects must be considered when comparing data and theory:

- The gluon distribution of the proton in this kinematic region is not well known. In Fig. 3a the data are compared with LO cross section curves [11] calculated using the 
GRV LO, CTEQ2M [24], MRSA [25] and MRSD' parton distribution sets for the proton. The MRSA and CTEQ2M parton distribution sets are global fits to data which include HERA measurements of the structure function $F_{2}$ [26]. The contribution from the tail of the LO resolved cross section with $x_{\gamma}^{L O} \geq 0.75$ is included in the calculations using the GS2 [27] photon parton distribution set. This contribution to the LO cross section is small $(d \sigma / d \bar{\eta} \approx 0.1 \mathrm{nb})$. This is also the case using other available photon parton distribution sets except for the LAC3 set [28], where due to the high gluon density at high $x_{\gamma}^{L O}$ the cross section becomes large enough to describe the whole high $x_{\gamma}^{O B S}$ cross section without any direct component. This parton distribution set is disfavoured by results presented in this paper (see below) and by other measurements [8].

- QCD calculations of this cross section are only available at LO. Some estimate of the size of higher order corrections can be made by comparing the LO calculation using the GRV LO parton distribution set with the other LO calculations using NLO parton distribution sets, which in general are around $20 \%$ lowerf. In addition, using $2 E_{T}^{\text {jet }}$ for the hard scale instead of $E_{T}^{\text {jet }} / 2$ lowers the cross section by up to $20 \%$. When both jets are at equal $\eta$ and equal $E_{T}^{j e t}, \sqrt{\hat{s}}=2 E_{T}^{j e t}$ and so the most common choices of scale are covered by the range $E_{T}^{\text {jet }} / 2$ to $2 E_{T}^{\text {jet }}$.

- Due to the fact that we are probing low $x$ partons in the proton, the standard approximation that the incoming partons are collinear and on-shell may be invalid. Analytic estimates of the effect of allowing the incoming partons to develop non-zero transverse momentum $\left(k_{T}\right)$ have been made in [29] using a so-called ' $k_{T}$ factorization' prescription. Calculations of the cross section using this technique are so far available only for the photon-gluon fusion contribution to the direct photon cross section. The typical $k_{T}$ developed is of the order of a GeV. In Fig. $3 \mathrm{~b}$ we show again the data of Fig. 3a, and the standard LO QCD calculation using the GRV LO proton parton distributions. Also shown are the curves for the same parton distribution set for the $x_{\gamma}^{L O}=1$ contribution alone, and for just the gluon-induced part of the $x_{\gamma}^{L O}=1$ cross section. (From this it can be seen that according to LO QCD, most of the direct photon cross section is attributable to the photon-gluon fusion diagram.) This latter curve may then be compared to the final curve, which shows the ' $k_{T}$ ' result for the same cross section. Non-zero parton $k_{T}$ in this prescription lowers the cross section by as much as $30 \%$, and could bring a full calculation into better agreement with the data.

- Non-perturbative 'hadronisation' effects can be expected to be significant for jets. In Fig. 3re we show the data of Fig. Ba compared to Monte Carlo estimates of the cross section calculated from partons generated with HERWIG using the GRV LO proton parton distribution set and the LAC1 [28] photon parton distribution set. Also shown is the cross section obtained by performing jet finding on the simulated final state particles. This histogram agrees with the shape of the data better than the analytic LO QCD calculations of Fig. Ba. Over most of the $\bar{\eta}$ region these histograms lie within $20 \%$ of each other.

Considering the uncertainties in LO calculations arising from the choice of the hard scale (taken to be $E_{T}^{j e t} / 2$ in these calculations) and the fact that hadronisation effects are not included

\footnotetext{
${ }^{2}$ The curve calculated with the GRV NLO parton distribution set (not shown) is also below the GRV LO curve by around $20 \%$.
} 
in the theoretical curves, the description of the measured points by the theoretical curves is reasonable. The effects of $k_{T}$, hadronisation, and of using different parton distributions are of comparable magnitude. That the direct jet cross section is sensitive to the parton densities of the proton can be seen by comparing different Monte Carlo estimates of this curve generated using different proton parton distribution sets. In Fig. 3 $\mathrm{d}$, we show the data compared to hadronic jet cross sections estimated with HERWIG using the GRV LO and MRSD 0 proton parton distribution sets. The LAC1 photon parton distribution set was used in both cases

for the LO resolved $x_{\gamma}^{O B S} \geq 0.75$ contribution. The separation between parton distribution sets remains after hadronisation, and the $\mathrm{MRSD}_{0}$ parton distribution gives a consistently lower cross section for negative values of $\bar{\eta}$.

In summary, the LO QCD predictions for the direct cross section are consistent with the data at the level of $30 \%$. The theoretical cross section is sensitive to the choice of proton parton distribution function. Complete NLO calculations will reduce the ambiguity in comparisons to the data.

\subsection{Resolved photon dijet cross section}

The acceptance correction for the resolved photon process as defined in Section 2 with a cut of $x_{\gamma}^{O B S}<0.75$ has been evaluated in the same way as in the direct photon cross section measurement. The efficiency and purity are now evaluated for bins with centres in the range $0.0<\bar{\eta}<1.5$. The efficiency and purity are both approximately flat and around $40 \%$, giving an acceptance correction factor of around unity across the whole range of $\bar{\eta}$. As for the direct measurement, the purity depends mostly on migrations across the $E_{T}^{j e t}$ cut. No subtraction of jet pedestal energy is carried out. However, variations in the kinematic selection of the analysis now give systematic variations in the cross section which are larger than the statistical errors, and thus the systematic error bars are correspondingly larger. As was seen in Fig. 2 $c$ c, both Monte Carlo simulations fail to describe the forward region of the transverse energy flow around the jet axis, and a reduction of these systematic uncertainties will require improvements in the simulations used. In Fig. 田 the correlated uncertainty arising from the description of the calorimeter energy response in the Monte Carlo is again shown separately by the shaded band. The band also includes the $3.3 \%$ uncertainty in the measurement of the integrated luminosity. The shaded band represents the width of the uncertainty around each data point.

Table 2 and Fig. 4 shows the measured cross section $d \sigma / d \bar{\eta}$ for $x_{\gamma}^{O B S}<0.75$ and the kinematic range indicated in the figure caption. The cross section is around $2 \mathrm{nb}$ for central values of $\bar{\eta}$ and rises to $4 \mathrm{nb}$ at $\bar{\eta}=1.5$. The LO cross sections, shown for comparison, are calculated using different photon parton distribution sets and the MRSA set for the proton. The theoretical sensitivity to the parton distributions in the proton is small (not shown), with the variations between curves calculated using different parton distribution sets being much less than the estimated errors on the measured cross section. As $x_{p}$ is higher in these events than in the direct, the effect of $k_{T}$ from partons in the proton on this calculation is expected to be small.

The DG [30], GRV LO and GS2 parton distribution sets reproduce the shape of the cross section well and can be brought into agreement with the data by applying a multiplicative factor of 1.5 to 2. (Note that the available NLO calculations [31] for inclusive jet photoproduction, which is dominated by the resolved process, differ from LO calculations by a factor of up to two.) The LO calculations using the LAC1 and LAC3 parton distribution sets cannot be brought into 


\begin{tabular}{|c|c|c|c|c|}
\hline $\bar{\eta}$ & $\begin{array}{c}d \sigma / d \bar{\eta} \\
(\mathrm{nb})\end{array}$ & $\begin{array}{c}\text { Statistical } \\
\text { Error }(\mathrm{nb})\end{array}$ & $\begin{array}{c}\text { Systematic } \\
\text { Error }(\mathrm{nb})\end{array}$ & $\begin{array}{c}\text { Energy Scale Uncertainty } \\
(\mathrm{nb})\end{array}$ \\
\hline 0.00 & 1.74 & 0.10 & 0.22 & 0.66 \\
0.25 & 2.20 & 0.13 & 0.23 & 0.54 \\
0.50 & 2.64 & 0.13 & 0.33 & 0.59 \\
0.75 & 3.51 & 0.16 & 0.51 & 0.71 \\
1.00 & 3.83 & 0.17 & 0.63 & 0.66 \\
1.25 & 3.88 & 0.16 & 0.51 & 0.91 \\
1.50 & 4.32 & 0.16 & 0.66 & 0.99 \\
\hline
\end{tabular}

Table 2: $d \sigma / d \bar{\eta}$ for $e p \rightarrow e X+2$ (or more) jets, $|\Delta \eta|<0.5, E_{T}^{j e t}>6 \mathrm{GeV}, 0.2<y<0.8$, $Q^{2}<4 \mathrm{GeV}^{2}, x_{\gamma}^{O B S}<0.75$.

agreement with the data by a constant normalisation factor. However, since the jet pedestal energies are not well described by the Monte Carlo for the resolved process, it is difficult to estimate what the effects of hadronisation and parton showering might be on the shapes of the curves, and so no parton distribution set can be completely excluded at this stage, although LAC3 is disfavoured.

\section{Conclusions}

Differential dijet cross sections have been measured in photoproduction with the ZEUS detector at HERA. The cross sections measured are $d \sigma / d \bar{\eta},|\Delta \eta|<0.5, E_{T}^{\text {jet }}>6 \mathrm{GeV}$ and $0.2<$ $y<0.8$, in the regions of $x_{\gamma}^{O B S} \geq 0.75$ (direct photoproduction) and $x_{\gamma}^{O B S}<0.75$ (resolved photoproduction). The measured cross sections have been defined in such a way that they are calculable to higher orders in QCD. We have corrected back to the final state particles and no subtraction of jet pedestal energies has been carried out. Our results are compared with the expectations of LO QCD. The direct cross section, which is sensitive to the gluon content of the proton, is consistent with LO QCD calculations to within 30\%. Hadronisation, incoming parton $k_{T}$ and the choice of scale also influence this comparison. The shape of the resolved cross section, which is sensitive to the gluon content of the photon, is described by LO QCD calculations using the DG, GRV LO and GS2 photon parton distributions. However, most LO QCD calculations lie below the data by a factor of 1.5 to 2. Comparison with NLO calculations for both the direct and resolved photon cross sections would be extremely valuable and should allow stronger conclusions to be drawn.

\section{Acknowledgements}

We thank the DESY Directorate for their strong support and encouragement and the HERA machine group for providing colliding beams. We acknowledge the assistance of the DESY computing and networking staff. It is also a pleasure to thank J. R. Forshaw and R. G. Roberts for useful discussions and for providing calculations based upon their publications. 


\section{References}

[1] H1 Collab., T. Ahmed et al., Phys. Lett. B297 (1992) 205.

[2] ZEUS Collab., M. Derrick et al., Phys. Lett. B297 (1992) 404.

[3] H1 Collab., I. Abt et al., Phys. Lett. B314 (1993) 436.

[4] ZEUS Collab., M. Derrick et al., Phys. Lett. B322 (1994) 287.

[5] ZEUS Collab., M. Derrick et al., DESY 94-176, accepted for publication by Phys. Lett. B.

[6] ZEUS Collab., M. Derrick et al., DESY 94-192, accepted for publication by Phys. Lett. B; H1 Collab., I. Abt et al., Phys.Letters B321 (1994) 161.

[7] For recent reviews, see Proc. of the Workshop on Two-Photon Physics at LEP and HERA, Lund, May 26-28, 1994.

[8] AMY Collab., R. Tanaka et al., Phys. Lett. B277 (1992) 215;

B. J. Kim et al., Phys. Lett. B325 (1994) 248.

[9] TOPAZ Collab., R. Enemoto et al., Phys. Lett. B328 (1994) 535.

[10] G. D'Agostini and D. Monaldi, Proc. of the Workshop on Physics at HERA Vol. 1 (1991) p527; A. Valkarova ibid p535.

[11] J. R. Forshaw and R. G. Roberts, Phys. Lett. B319 (1993) 539.

[12] ZEUS Collab., The ZEUS Detector, Status Report (1993).

[13] M. Derrick et al., Nuc. Instr. Meth. A 309 (1991) 77;

A. Andresen et al., Nuc. Instr. Meth. A 309 (1991) 101;

A. Bernstein et al., Nuc. Instr. Meth. A 336 (1993) 23.

[14] C. Alvisi et al., Nuc. Instr. Meth. A305 (1991) 30.

[15] B. Foster et al., Nuc. Phys. B, Proc. Suppl. B32 (1993) 181.

[16] J. Andruszków et al., DESY 92-066 (1992).

[17] ZEUS Collab., M. Derrick et al., Z. Phys. C65 (1995) 379.

[18] J. Huth et al., Proc. of the 1990 DPF Summer Study on High Energy Physics, Snowmass, Colorado, edited by E.L. Berger (World Scientific, Singapore,1992) p. 134.

UA1 Collab., G. Arnison et al., Phys. Lett. 123B (1983) 115.

[19] G. Marchesini et al., Comp. Phys. Comm. 67 (1992) 465.

[20] H.-U. Bengtsson and T. Sjöstrand, Comp. Phys. Comm. 46 (1987) 43.

T. Sjöstrand, CERN-TH.6488/92.

[21] A. Martin, W.J. Stirling and R.G. Roberts, Phys. Rev. D47 (1993) 867.

[22] M. Glück, E. Reya and A. Vogt, Phys. Rev. D46 (1992) 1973. 
[23] G. D'Agostini, A Multidimensional Unfolding Method Based on Bayes' Theorem, DESY 94-099.

[24] W. Tung, Proc. of International Workshop on Deep Inelastic Scattering and Related Subjects, Eilat, Israel, 1994 World Sci., Singapore (to be published);

H. L. Lai et al., MSU-HEP-41024, CTEQ-404.

[25] A. Martin, W.J. Stirling and R.G. Roberts, Phys. Rev. D50 (1994) 6734.

[26] The CTEQ2 distributions use the final 1992 HERA data published in ZEUS Collab., M. Derrick et al., Phys. Lett. B316 (1993) 412; H1 Collab., I. Abt et al., Nucl. Phys. B407 (1993) 515.

MRSA uses the preliminary 1993 HERA data: ZEUS Collab., G. Wolf, Proc. of International Workshop on Deep Inelastic Scattering, Eilat, Israel, Feb. 1994; M. Roco, Proc. of 29th Rencontre de Moriond, March 1994 ; H1 Collab., K. Müller, ibid.

[27] L. E. Gordon and J. K. Storrow, Z. Phys. C56 (1992) 307.

[28] H. Abramowicz, K. Charchula and A. Levy, Phys. Lett. B269 (1991) 458.

[29] J. R. Forshaw and R. G. Roberts, Phys. Lett. B335 (1994) 494.

[30] M.Drees and K.Grassie, Z. Phys. C28 (1985) 451.

[31] G. Kramer and S.G. Salesch, Z. Phys. C61 (1994) 277;

L.E. Gordon and J.K. Storrow, Phys. Lett. B291 (1992) 320;

D. Bödeker, Z. Phys. C59 (1993) 501;

M. Greco and A. Vicini, Frascati preprint, LNF-93/017 (April 1993);

D. Bödeker, G. Kramer and S.G. Salesch, Z. Phys. C63 (1994) 471;

M. Klasen, G. Kramer and S.G. Salesch, DESY preprint DESY 94-232;

P. Aurenche et al., Phys. Lett. B338 (1994) 98. 


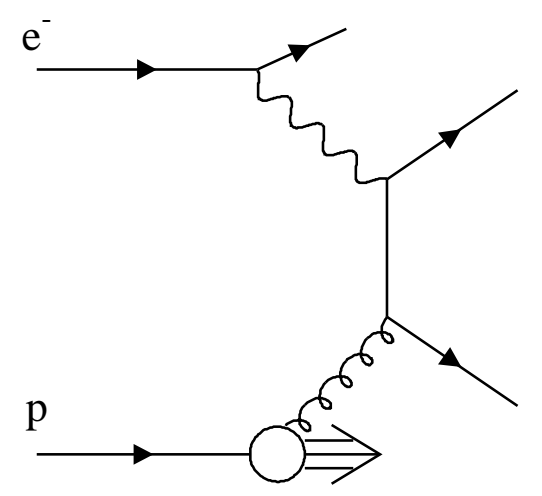

a)

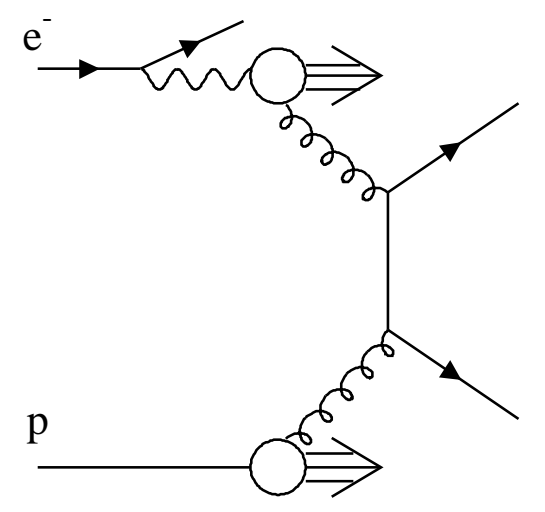

b)

Figure 1: Examples of leading order diagrams for a) direct and b) resolved photoproduction. 


\section{ZEUS 1993}
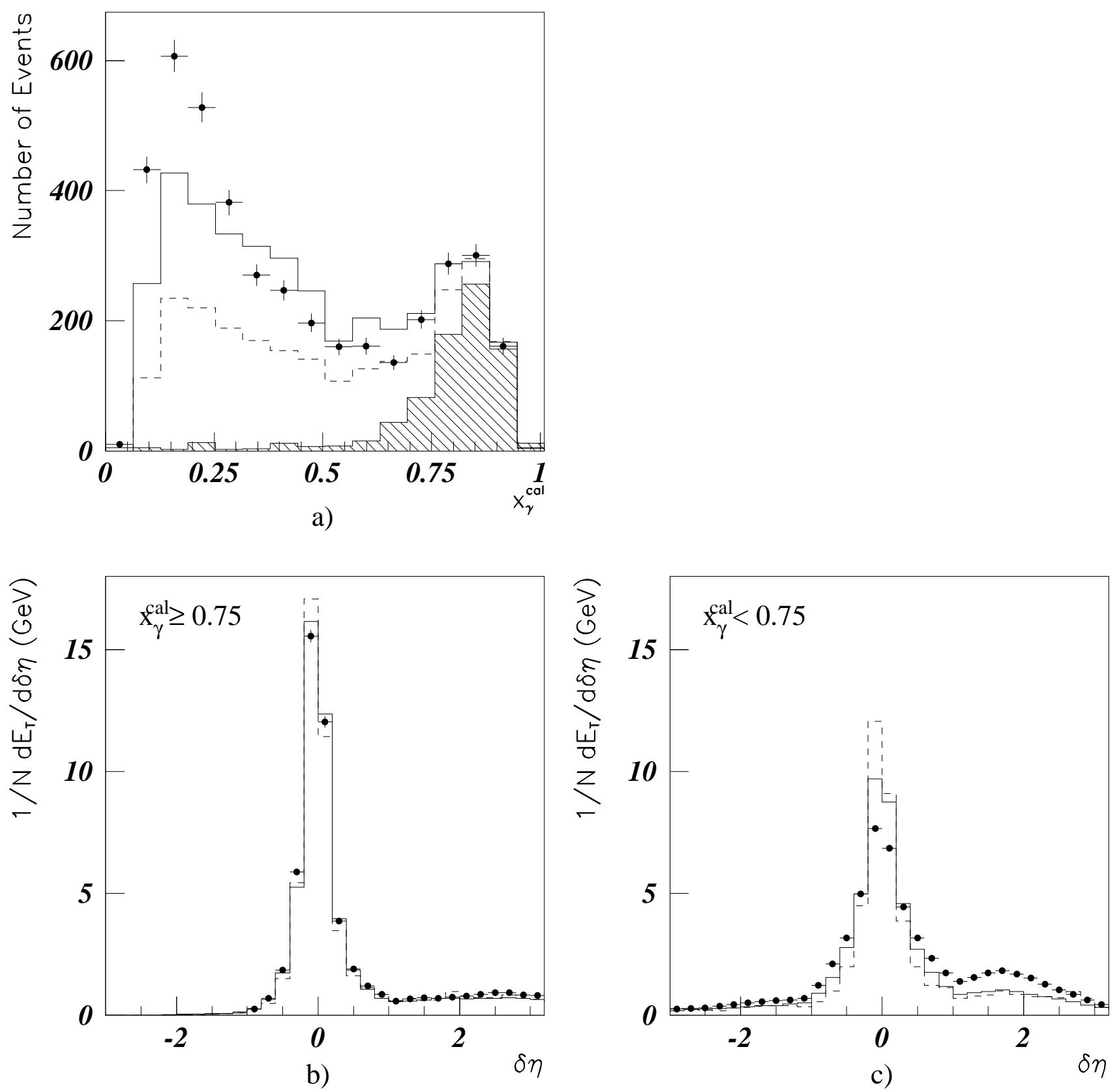

Figure 2: a) The $x_{\gamma}^{c a l}$ distribution. The solid circles are uncorrected ZEUS data. The solid (dashed) line represents the distribution from the PYTHIA (HERWIG) simulation. The LO direct contribution to the HERWIG distribution is shown by the shaded histogram. The Monte Carlo curves have been normalised to fit the direct peak in the data. b) and c) show the uncorrected transverse energy flow $1 / N d E_{T} / d \delta \eta$ around the jet axis, for cells within one radian in $\phi$ of the jet axis, for b) direct and c) resolved events. The solid (dashed) line represents the distribution from PYTHIA (HERWIG). 


\section{ZEUS 1993}

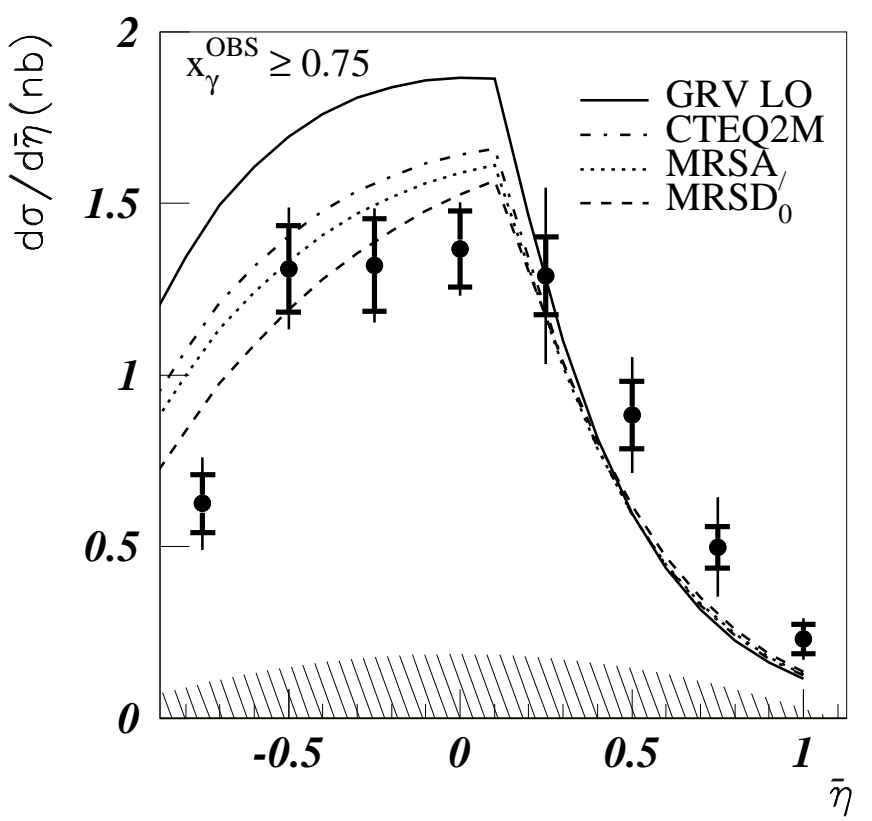

a)

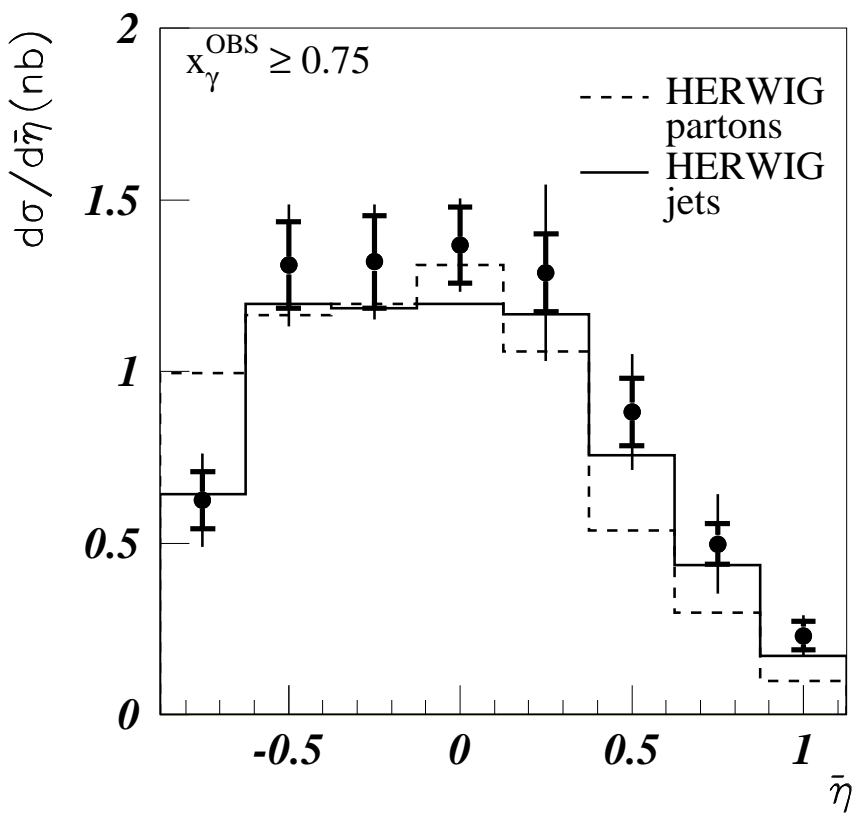

c)

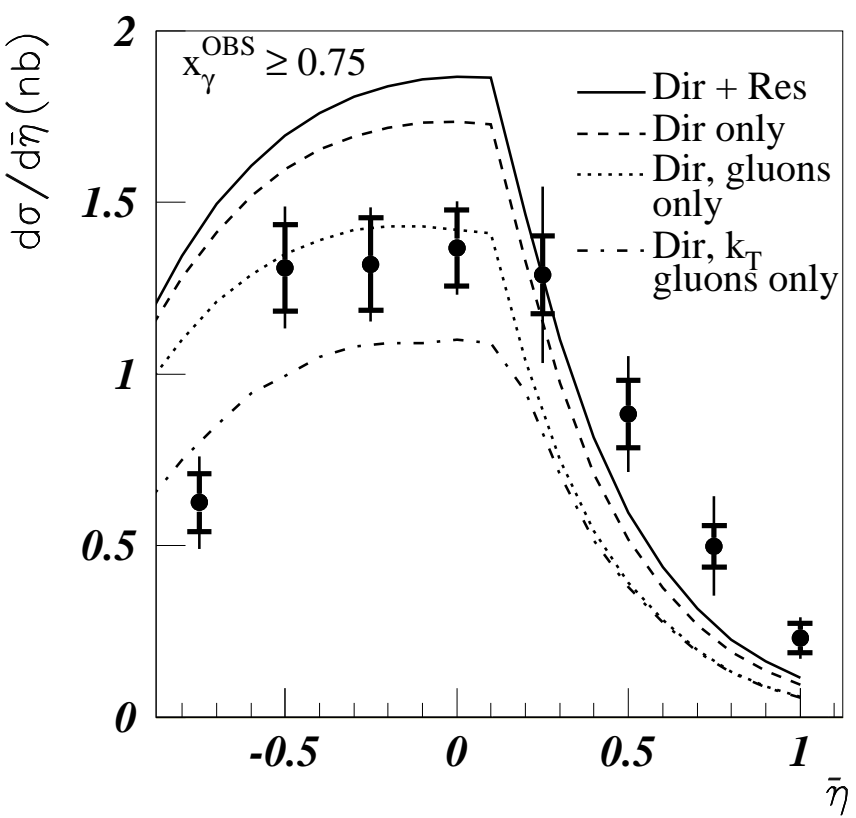

b)

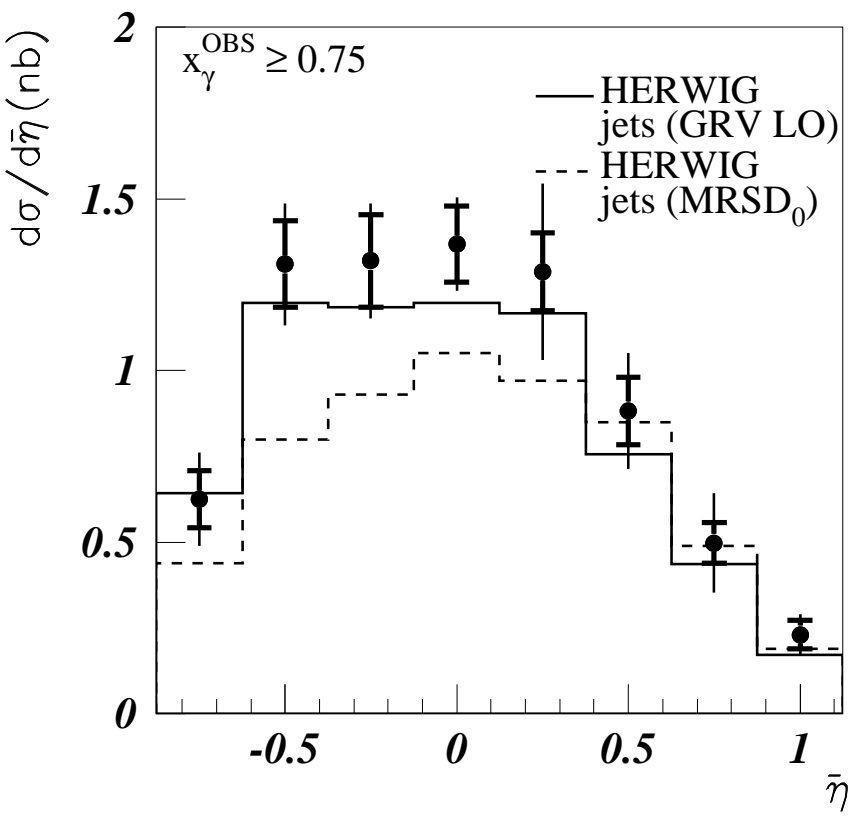

d)

Figure 3: $\quad d \sigma / d \bar{\eta}$ for $e p \rightarrow e X+2$ (or more) jets, $|\Delta \eta|<0.5, E_{T}^{\text {jet }}>6 \mathrm{GeV}, 0.2<y<$ $0.8, Q^{2}<4 \mathrm{GeV}^{2}, x_{\gamma}^{O B S} \geq 0.75$. The solid circles are corrected ZEUS data. The inner error bars indicate the statistical errors, the outer error bars show the systematic uncertainty (excluding the correlated uncertainty) added in quadrature. The shaded band shows the correlated uncertainty from measurement of energy in the calorimeter and the integrated luminosity. In a) the data are compared to LO QCD calculations using several parton distribution sets for the proton and the GS2 set for the photon. In b) the data are compared to the LO QCD calculation of a) using the GRV LO parton distribution set for the proton, the same calculation but omitting the resolved contribution with $x_{\gamma}^{L O} \geq 0.75$, including only gluon induced direct photon processes and the ' $k_{T}$ factorized' curve. In c) the data are compared to HERWIG Monte Carlo estimates of the cross section using partons and final state jets. For these HERWIG histograms we have used the the GRV LO (LAC1) proton (photon) parton distribution set. In d) the data are compared to HERWIG jet cross sections using the GRV (LAC1) and $\mathrm{MRSD}_{0}$ (LAC1) proton (photon) parton distribution sets. 


\section{ZEUS 1993}

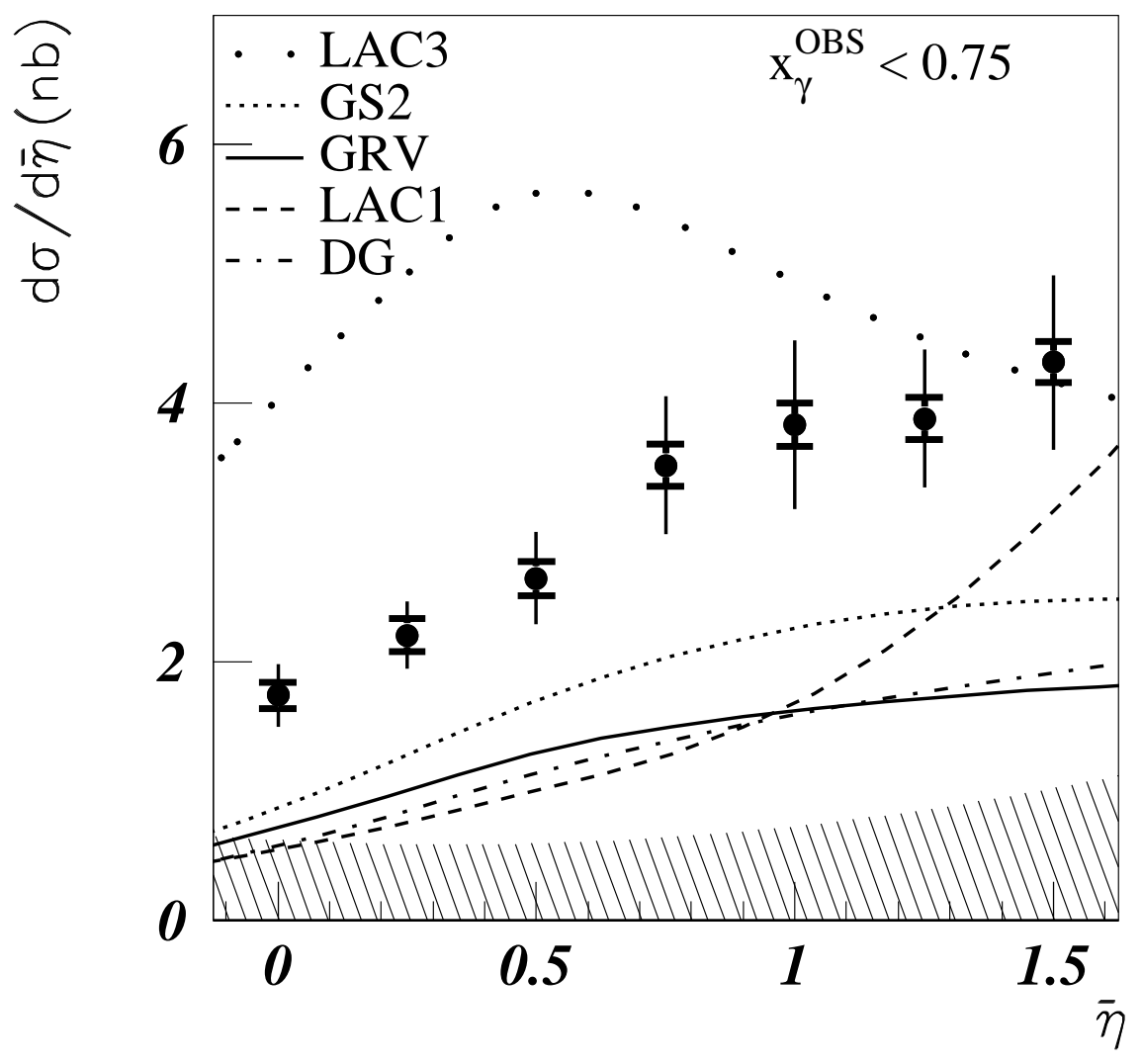

Figure 4: $\quad d \sigma / d \bar{\eta}$ for $e p \rightarrow e X+2$ (or more) jets, $|\Delta \eta|<0.5, E_{T}^{\text {jet }}>6 \mathrm{GeV}, 0.2<y<$ $0.8, Q^{2}<4 \mathrm{GeV}^{2}, x_{\gamma}^{O B S}<0.75$. The solid circles are corrected ZEUS data. The inner error bars indicate the statistical errors, the outer error bars show the systematic uncertainty (excluding the correlated uncertainty) added in quadrature. The shaded band shows the correlated uncertainty from measurement of energy in the calorimeter and the integrated luminosity. Also shown are LO QCD calculations. The parton distribution sets used for the photon are LAC3, GS2, GRV, LAC1 and DG. The proton parton distribution set used is the MRSA set. 